\title{
Multiscale quantification of thermal expansion of concrete and thermal stresses of concrete structures
}

\author{
H. Wang \& H.A. Mang \\ Institute for Mechanics of Materials and Structures, TU Wien-Vienna University of Technology, Vienna, Austria \\ College of Civil Engineering, Tongji University, Shanghai, China
}

Y. Yuan

College of Civil Engineering, Tongji University, Shanghai, China

B. Pichler

Institute for Mechanics of Materials and Structures, TU Wien-Vienna University of Technology, Vienna, Austria

\begin{abstract}
The mechanical behavior of concrete structures subjected to temperature changes is significantly influenced by hygro-thermal processes occurring at the nanoscopic material scale. In order to quantify this relation exemplarily, a multiscale structural analysis of a simply supported concrete beam subjected to sudden cooling at its top surface is carried out. The overall analysis is organized in three steps. The first step refers to upscaling of thermoelastic properties of concrete by means of a multiscale model. It uses measured "hygrothermic coefficients" as input. They quantify the change of the internal relative humidity resulting from a temperature change. The multiscale model links temperature-induced changes of effective pore pressures in nanoscopic gel and capillary pores to the macroscopic thermal expansion behavior of the cement paste and the concrete. In the present contribution, this upscaling approach is validated by comparing model-predicted thermal expansion coefficients of cement paste with measured counterparts. The second step of the overall analysis consists of a macroscopic thermoelastic Finite Element analysis of the aforementioned concrete beam. These simulations are based on the homogenized elastic stiffness and the homogenized thermal expansion coefficient of concrete obtained in the first step. The simulations deliver distributions of the temperature and of the macroscopic stresses inside the analyzed concrete beam. In the third step, the obtained macroscopic stresses of concrete and the corresponding temperature changes are downscaled to average stress states of the cement paste matrix and of the aggregate inclusions, respectively. This way, it is shown that the significant mismatch of the thermal expansion coefficients of cement paste and aggregates results in microscopic tensile stresses of cement paste, which are significantly larger than the macroscopic tensile stresses experienced by concrete.
\end{abstract}

\section{INTRODUCTION}

When steel-reinforced concrete structures are subjected to temperature changes, all material constituents exhibit thermal eigenstrains. Given that the thermal expansion coefficients of steel and concrete are similar, there is no significant mismatch of thermal eigenstrains. However, the concrete constituents "cement paste" and "aggregates" do exhibit significantly different thermal expansion properties.

- Concrete aggregates exhibit thermal expansion coefficients ranging between $4 \cdot 10^{-6} /{ }^{\circ} \mathrm{C}$ and $12 \cdot 10^{-6} /{ }^{\circ} \mathrm{C}$, mainly depending on the mineral composition.

- The cement paste exhibits thermal expansion coefficients ranging between $8 \cdot 10^{-6} /{ }^{\circ} \mathrm{C}^{-1}$ and
$24 \cdot 10^{-6} /{ }^{\circ} \mathrm{C}$, mainly depending on the internal relative humidity (Emanuel \& Hulsey 1977). This dependence is typically attributed to the experimentally measured increase of the internal relative humidity, $\Delta R H$, resulting from a temperature increase, $\Delta T$. In this context, the ratio $\Delta R H / \Delta T$ is referred to as the "hygrothermic coefficient".

The described experimental observations suggest that thermal expansion of concrete is a multiscale thermo-hygro-poro-mechanical problem. This calls for multiscale structural analysis of concrete structures, which combines multiscale modeling of concrete with structural simulations.

The multiscale model of concrete used in the present contribution reflects the hierarchical organization of concrete based on four different matrix- 
inclusion composites, introduced at four different scales of observation, see also (Wang et al. 2017b, Wang et al. submitted) and Figure 1. Concrete is considered to consist of a cement paste matrix and of spherical aggregate inclusions Figure 1.

The multiscale model renders two types of scale transition possible.

- Bottom-up homogenization of the elastic stiffness and of the thermal expansion properties of concretes.

- Top-down calculation of microscopic stress states inside the concrete constituents "cement paste" and "aggregates".

Both types of scale transitions turn out to be important for multiscale structural analysis of a thermally loaded concrete beam subjected to a sudden temperature change. This analysis is structured as follows:

1. An analytical solution of the heat conduction problem is presented. This involves a series solution of the temperature field history.

2. Thermo-mechanical Finite Element simulations of the concrete beam are performed. They use calculated temperature fields, the homogenized stiffness and the homogenized thermal expansion coefficient of concrete as input. The macroscopic simulations deliver thermal stresses in the homogenized concrete.

3. Microscopic stresses of cement pastes and aggregates are calculated based on the multiscale model, using the temperature field (from item 1) and the macroscopic concrete stresses (from item 2) as input.

The present contribution is organized as follows. Section 2 contains a brief overview over the multiscale model of concrete. Section ? deals with the multiscale structural analysis of a concrete beam subjected to a sudden temperature change. Section 3 contains conclusions.

\section{THERMO-HYGRO-PORO-MECHANICAL MULTISCALE MODEL OF CONCRETE}

\subsection{Modelling of gel and capillary pore spaces}

Both gel and capillary pores are considered to be spherical, with pore radii $r$ following exponential distributions (Huang et al. 2015)

$$
\phi_{k}^{p d f}(r)=\frac{1}{R_{k}} \exp \left(-\frac{r}{R_{k}}\right),
$$

with $k \in[$ gpor; cpor $]$. In Eq.(1), $R_{\text {gpor }}=12 \mathrm{~nm}$ and $R_{\text {cpor }}=12 \mathrm{~nm}$ denote the characteristic radius of the gel and the capillary pores, respectively (Wang et al. 2017b).

Considering partially saturated conditions, pores with radii smaller than $r_{l g}$ are filled by porewater, while larger pores are occupied by air. Thereby, $r_{l g}$ denotes the curvature of the menisci between liquidfilled and gas-filled domains. The curvature is a function of both the absolute temperature $T$ and the internal relative humidity $R H$, as described by the Kelvin-Cohan equation (Neimark \& Ravikovitch 2001).

$r_{l g}=\frac{2 \gamma^{l g} v_{m} \cos \theta}{\ln (R H) R T}+t$,

where $R, \gamma^{g}, \theta$, and $v_{m}$ denote the universal gas constant, the surface tension prevailing in a liquid-gas interface, the contact angle of porewater, and the molar volume of water, respectively. Also in Eq. (2), $t$ denotes the thickness of water layers which are adsorbed to the pore surfaces. It increases with increasing relative humidity, as quantified by Badmann et al. (1981),

$$
t=0.385 \mathrm{~nm}-\ln [-\ln (R H)] \cdot 0.189 \mathrm{~nm} .
$$

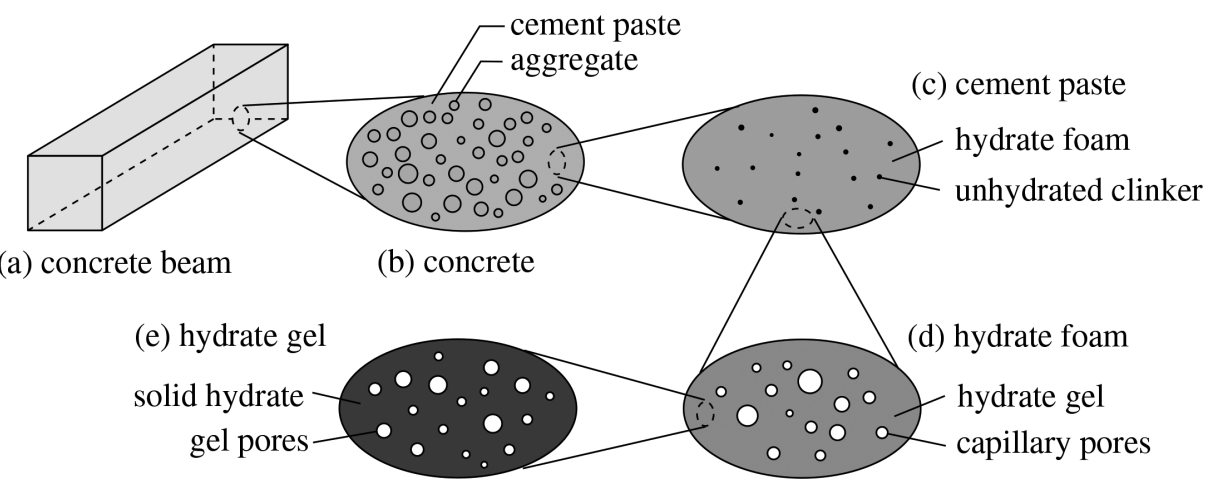

Figure 1. Multiscale representation of a concrete structure. 


\subsection{Effective pore pressures relevant for homogenization}

The solid skeleton around the pores is subjected to so-called "effective pore pressures", combining the influence of the fluid pressure and of the surface tension. Considering that porewater is a perfectly wetting fluid, the effective pore pressures $\mathrm{p}(\mathrm{r})$ can be written as (Wang et al. 2017b)

$$
p(r)=\left\{\begin{array}{cll}
\ln (R H) \frac{R T}{v m} & \cdots & r \leq r_{i g}, \\
-\frac{2 \gamma^{l g}}{r-t} & \cdots & r>r_{i g} .
\end{array}\right.
$$

Average effective pore pressures of gel and capillary pores, $p_{g p o r}$ and $p_{c p o r}$, are relevant for upscaling of the pore-size-dependent effective pressures, see Eq. (4), to the material scale of concrete, see (Pichler \& Dormieux 2010). With reference to Eq. (1), they read as

$$
p_{k}=\frac{1}{R_{k}} \int_{0}^{\infty} p(r) \exp \left(-\frac{r}{R_{k}}\right) \mathrm{d} r,
$$

with $k \in$ [gpor; cpor].

\subsection{Further phase input for homogenization}

Homogenization of the thermoelastic properties of the matrix-inclusion composites shown in Figure 1 is carried out in the framework of the Mori-Tanaka scheme. Each representative volume element (RVE), V, is subdivided into a matrix phase and an inclusion phase, occupying the domains $V_{m}$ and $V_{i}$, respectively, where $m$ stands for "matrix" and $i$ for "inclusion". Both material phases $k \in[m ; i]$ exhibit a specific elastic stiffness $C_{k}$ and a specific eigenstress $\boldsymbol{\sigma}_{k}^{\iota}$,

$\forall \underline{x} \in V_{k}:\left\{\begin{array}{l}\mathbb{C}(\underline{x})=\mathbb{C}_{k}, \\ \boldsymbol{\sigma}^{e}(\underline{x})=\boldsymbol{\sigma}_{k}^{e},\end{array}\right.$

as well as a specific volume fraction $f_{k}$, given as

$$
f_{k}=\frac{V_{k}}{V} \text {. }
$$

The phase stiffness tensors $C_{k}$, the phase eigenstress tensors $\boldsymbol{\sigma}_{k}^{e}$, and the phase volume fractions $f_{k}$, are described in the following three paragraphs.

The elastic stiffness tensors $\mathbb{C}_{k}$ of all material phases of concrete are considered to be isotropic. Therefore, they may be expressed in terms of phase bulk moduli, $k$, and phase shear moduli, $\mu$, (see Table 1):

$\mathbb{C}_{k}=3 k_{k} \mathrm{II}_{v o l}+2 \mu_{k} \mathrm{II}$ dev.

In Eq. (8), $\mathrm{II}_{v o l}$ and $\mathrm{II}_{d e v}$ are the volumetric and the deviatoric part, respectively, of the symmetric fourthorder identity tensor II, i.e. II $=\mathrm{II}_{v o l}+I_{d e v}$, see, e.g. (Wang et al. 2017b) for more details.

The eigenstress tensors $\boldsymbol{\sigma}_{k}^{\iota}$ of the pore phases are equal to the effective pore pressures defined in Eq. (5)

$$
\boldsymbol{\sigma}_{k}^{e}=-p k^{1},
$$

with $k \in$ [gpor; cpor]. As for the solid phases, the eigenstresses are induced by the thermal eigenstrains

$$
\boldsymbol{\sigma}_{k}^{e}=-\mathbb{C}_{k}: \mathcal{E}_{k}^{e}=-\mathbb{C}_{k}: \alpha_{k} \Delta T \mathbf{1},
$$

with $k \in[h y d ; c e m ; a g g]$.

The volume fractions of the concrete constituents are functions (i) of the initial composition, quantified in terms of the aggregate-to-cement mass ratio $a / c$ and the initial water-to-cement mass ratio $w / c$, and (ii) of the maturity of the material, quantified in terms of the hydration degree $\xi$. At the concrete scale, the volume fractions of the cement paste and the aggregates read as (Pichler \& Hellmich 2011):

$$
f_{c p}^{c o n}=\frac{\frac{a / c}{\rho a g g}}{\frac{1}{\rho_{c e m}}+\frac{w / c}{\rho \mathrm{H}_{2} \mathrm{O}}+\frac{a / c}{\rho_{a g g}}}, \quad f_{a g g}^{c o n}=1-f_{c p}^{c o n}
$$

where $\rho_{\text {agg }}=2648 \mathrm{~kg} / \mathrm{m}^{3}, \rho_{\text {cem }}=3150 \mathrm{~kg} / \mathrm{m}^{3}$, and $\rho \mathrm{H}_{2} \mathrm{O}=1000 \mathrm{~kg} / \mathrm{m}^{3}$ are the mass densities of aggregates, cement, and water, respectively. At the cement paste scale, the volume fractions of the hydrate foam and the residual cement grains read as (Pichler \& Hellmich 2011):

Table 1. Bulk and shear moduli of the material phases of concrete.

\begin{tabular}{llll}
\hline Phase & Bulk modulus $k[\mathrm{GPa}]$ & Shear modulus $\mu[\mathrm{GPa}]$ & Sources \\
\hline Solid hydrate & 31.8 & 19.1 & Ulm et al. (2004) \\
Cement & 116.7 & 53.8 & Pichler \& Hellmich (2011) \\
Aggregate & 22.2 & 16.7 & Gudmundsson (2011) \\
\hline
\end{tabular}


$f_{h f}^{c p}=\frac{w / c+0.32 \xi}{w / c+0.32}, \quad f_{c e m}^{c p}=1-f_{h f}^{c p}$.

At the hydrate foam scale, the volume fractions of the hydrate gel and the capillary pores read as (Pichler \& Hellmich 2011):

$f_{g e l}^{h f}=\frac{0.68 \xi}{w / c+0.32 \xi}, \quad f_{c p o r}^{h f}=1-f_{g e l}^{h f}$.

At the hydrate gel scale, the volume fractions of the solid hydrates and the gel pores read as (Ulm et al. 2004):

$f_{\text {hyd }}^{\text {gel }}=0.72, \quad f_{\text {gpor }}^{\text {gel }}=1-f_{\text {hyd }}^{g e l}$.

\subsection{Homogenization of thermoelastic properties of all involved matrix-inclusion composites}

The elastic behavior of any matrix inclusion composite can be expressed by means of the generalized Hooke's law as

$\sum_{\text {hom }}=\mathbb{C}_{\text {hom }}: \mathrm{E}_{\text {hom }}+\sum_{\text {hom }}^{e}$,

with $\Sigma_{\text {hom }}$ denoting the homogenized stress tensor, $\mathbb{C}_{\text {hom }}$ standing for the homogenized stiffness tensor, $\mathrm{E}_{\mathrm{hom}}$ denoting the homogenized strain tensor, and $\sum_{\text {hom }}^{e}$ standing for the homogenized eigenstress tensor. The homogenized stiffness tensor reads as (Hill 1963)

$\mathbb{C}_{\text {lom }}=f_{m} \mathbb{C}_{m}: \mathbb{A}_{m}+f_{i} \mathbb{C}_{i}: \mathbb{A}_{i}$

The homogenized eigenstress tensor reads as (Levin 1967)

$\sum_{\text {hom }}^{e}=f_{m} \boldsymbol{\sigma}_{m}^{e}: \mathbb{A}_{m}+f_{i} \boldsymbol{\sigma}_{i}^{\iota}: \mathbb{A}_{i}$.

Thereby, $\mathbb{A}_{m}$ and $\mathbb{A}_{i}$ are the phase strain concentration tensors of the matrix and of the inclusions, respectively. The corresponding MoriTanaka estimates read as (Mori \& Tanaka 1973, Benveniste 1987)

$$
\begin{aligned}
& \mathbb{A}_{k}=\left[\mathrm{II}+\mathbb{P}:\left(\mathbb{C}_{k}-\mathbb{C}_{m}\right]^{-1}\right. \\
& :\left[\sum_{j=m, i} f_{j}\left[\mathrm{II}+\mathbb{P}:\left(\mathbb{C}_{j}-\mathbb{C}_{m}\right)\right]^{-1}\right]^{-1}, k \in[m ; i],
\end{aligned}
$$

with $\mathbb{P}$ as the fourth-order Hill tensor for spherical phases.

As for the homogenization of the thermal expansion coefficient, $\alpha_{\text {hom }}$, the composite is subjected to temperature change $\Delta T$, and it is allowed to deform freely, such that the macroscopic stress tensor vanishes, i.e. $\Sigma_{\text {hom }}=0$. In such a scenario, the homogenized strain tensor $\mathrm{E}_{\mathrm{hom}}$ is equal to the homogenized thermal expansion coefficient $\alpha_{\text {hom }}$ multiplied by the second-order identity tensor 1, i.e.

$$
\mathrm{E}_{\mathrm{hom}}=\mathrm{E}_{\mathrm{hom}}^{\mathrm{e}}=-\mathbb{C}_{\mathrm{hom}}^{-1}: \sum_{\mathrm{hom}}^{e}=\alpha_{\mathrm{hom}} \Delta T \mathbf{1} .
$$

By inserting Eqs. (16) and (17) into Eq. (19), the homogenized thermal expansion coefficient of the matrix-inclusion composite can be determined.

\subsection{Bottom-up homogenization of concrete}

Analytical upscaling of the elastic stiffness of concrete, see Eq. (16), and of its eigenstress tensor, see Eq. (17), is carried in four subsequent steps, starting with the hydrate gel scale and continuing with the hydrate foam scale, the cement paste scale, and, finally, with the concrete scale, see Table 2.

As for the exemplary validation of these developments, model-predicted thermal expansion coefficients of mature cement pastes are compared with corresponding measurements. Hygrothermic coefficients, measured by Grasley \& Lange (2007), see Figure 2, together with the initial water-tocement mass ratios of the tested cement pastes serve as input for the multiscale model. The modelpredicted thermal expansion coefficients of cement pastes agree quite well with independent measurements, also reported by Grasley \& Lange (2007), see Figure 3 . This underlines the satisfactory performance of the developed multiscale model.

\subsection{Top-down stress concentration}

After a structural simulation, the macroscopic stress tensors of concrete, $\Sigma_{\text {con }}$, and the temperature changes $\Delta T$ are known at every point of the structure. Top-down stress concentration aims at quantifying microscopic stress states inside both the cement paste matrix and the aggregate inclusions.

This task is accomplished by explicit consideration of the underlying Eshelby problems. At first, the macrostrain is calculated from the macrostress and the temperature change as

$$
\mathrm{E}_{c o n}=\mathbb{C}_{c o n}^{-1}: \sum_{c o n}+\alpha_{c o n} \Delta T \mathbf{1} .
$$

The macrostrain allows for quantification of the auxiliary strain $\mathrm{E}_{\infty}$, representing the remote loading of the underlying Eshelby problems (Zaoui 2002).

$$
\begin{gathered}
\mathrm{E}_{\infty}=\left\{f_{c p} \mathrm{II}+f_{a g g}\left[\mathrm{II}+\mathbb{P}:\left(\mathbb{C}_{a g g}-\mathbb{C}_{c p}\right)\right]^{-1}\right\}^{-1} \\
:\left\{\mathrm{E}_{c o n}+f_{\text {agg }}\left[\mathrm{II}+\mathbb{P}:\left(\mathbb{C}_{a g g}-\mathbb{C}_{c p}\right)\right]^{-1}\right. \\
\left.: \mathbb{P}:\left(\boldsymbol{\sigma}_{a g g}^{e}-\boldsymbol{\sigma}_{\text {agg }}^{e}\right)\right\} .
\end{gathered}
$$


Table 2. Input and outputs of the step-by-step homogenization of the concrete.

\begin{tabular}{|c|c|c|c|c|c|c|}
\hline \multirow[b]{2}{*}{ RVE } & \multirow[b]{2}{*}{ Constituent } & \multirow[b]{2}{*}{ Volume fraction } & \multirow[b]{2}{*}{ Stiffness } & \multirow[b]{2}{*}{ Eigenstress } & \multicolumn{2}{|c|}{ Homogenized } \\
\hline & & & & & Stiffness & Eigenstress \\
\hline \multirow[t]{2}{*}{$\begin{array}{c}\text { hydrate } \\
\text { gel }\end{array}$} & solid hydrates & $f_{\text {hyd }}^{g e l}$ & $\mathbb{C}_{h y d}$ & $-\mathbb{C}_{h y d}: \alpha_{h y d} \Delta T 1$ & $\mathbb{C}_{\text {gel }}$ & $\sum_{g e l}^{e}$ \\
\hline & gel pores & $f_{\text {gpor }}^{\text {gel }}$ & 0 & $-p_{g p o r} 1$ & & \\
\hline \multirow[t]{2}{*}{$\begin{array}{l}\text { hydrate } \\
\text { foam }\end{array}$} & hydrate gel & $f_{g e l}^{h f}$ & $\mathbb{C}_{g e l}$ & $\sum_{g e l}^{e}$ & $\mathbb{C}_{h f}$ & $\sum_{h f}^{e}$ \\
\hline & capillary pores & $f_{\text {cpor }}^{h f}$ & 0 & $-p_{g p o r} 1$ & & \\
\hline \multirow[t]{2}{*}{$\begin{array}{r}\text { cement } \\
\text { paste }\end{array}$} & hydrate foam & $f_{h f}^{c p}$ & $\mathbb{C}_{h f}$ & $\sum_{h f}^{e}$ & $\mathbb{C}_{c p}$ & $\sum_{c p}^{e}$ \\
\hline & cement & $f_{c e m}^{c p}$ & $\mathbb{C}_{c e m}$ & $-\mathbb{C}_{c e m}: \alpha_{c e m} \Delta T 1$ & & \\
\hline \multirow[t]{2}{*}{ concrete } & cement paste & $f_{c p}^{c o n}$ & $\mathbb{C}_{c p}$ & $\sum_{c p}^{e}$ & $\mathbb{C}_{c o n}$ & $\sum_{c o m}^{e}$ \\
\hline & aggregates & $f_{a g g}^{c o n}$ & $\mathbb{C}_{\text {agg }}$ & $-\mathbb{C}_{a g g}: \alpha_{a g g} \Delta T 1$ & & \\
\hline
\end{tabular}

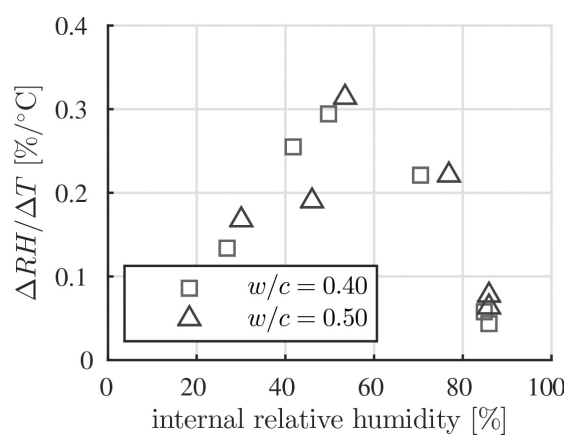

Figure 2. Experimentally determined hygrothermic coefficients $\Delta R H / \Delta T$ by Grasley \& Lange (2007) for mature cement pastes with an initial water-to-cement mass ratio $w / \mathrm{c} \in[0: 40 ; 0: 50]$.

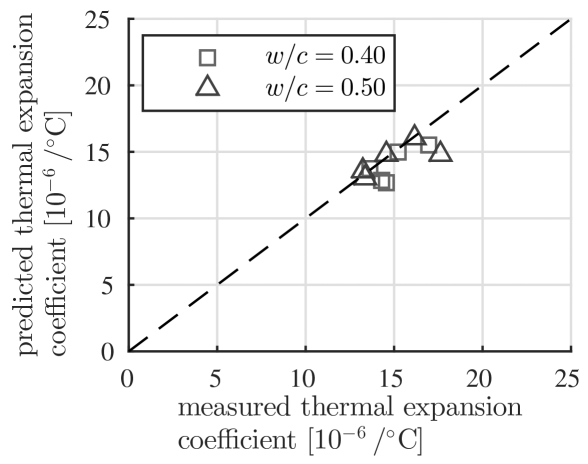

Figure 3. Comparison of model-predicted and measured thermal expansion coefficients of the cement paste with initial water-tocement mass ratios w/c $\in[0.40 ; 0.50 ;]$ the hydration degrees $\xi$ of the mature pastes are set equal to $80 \%$.
The microscopic strains of the cement paste and of the aggregates, $\boldsymbol{\sigma}_{c p}$ and $\boldsymbol{\sigma}_{a g g}$, are quantified as (Zaoui 2002)

$$
\begin{aligned}
\varepsilon_{c p}= & \mathrm{E}_{\infty}, \\
\varepsilon_{a g g} & =\left[\mathrm{II}+\mathbb{P}:\left(\mathbb{C}_{a g g}-\mathbb{C}_{c p}\right)\right] \\
& :\left[\mathrm{E}_{\infty}-\mathbb{P}:\left(\boldsymbol{\sigma}_{a g g}^{e}-\boldsymbol{\sigma}_{a g g}^{e}\right)\right] .
\end{aligned}
$$

The microscopic stresses of the cement paste and of the aggregates, $\boldsymbol{\sigma}_{c p}$ and $\boldsymbol{\sigma}_{a g g}$, finally follow as

$$
\begin{aligned}
& \boldsymbol{\sigma}_{c p}=\mathbb{C}_{c p}:\left(\mathcal{E}_{c p}-\mathcal{E}_{c p}^{\varepsilon}\right), \\
& \boldsymbol{\sigma}_{\text {agg }}=\mathbb{C}_{\text {agg }}:\left(\mathcal{E}_{\text {agg }}-\mathcal{E}_{\text {agg }}^{e}\right) .
\end{aligned}
$$

\section{MULTISCALE STRUCTURAL ANALYSIS OF A CONCRETE BEAM SUBJECTED TO SUDDEN COOLING AT THE TOP SURFACE}

The object of the following multiscale thermo mechanical structural analysis is a simply-supported concrete beam with a rectangular cross section, see Figure 4. In the initial configuration, the beam exhibits a uniform reference temperature Tref

$T(z, t<0)=T_{r e f}$.

At time instant $t=0$, the top surface of the beam is considered to be flooded by ice-water. 


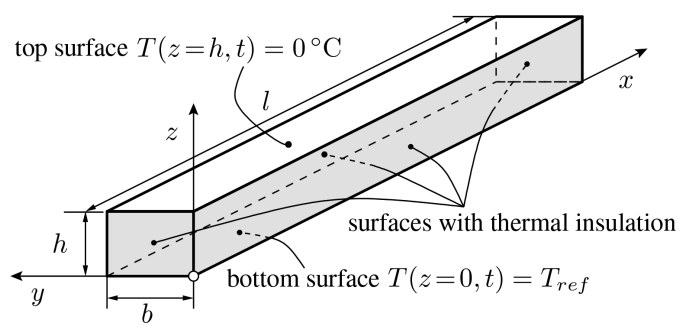

Figure 4. One-dimensional heat conduction along the height of a simply supported concrete beam with a rectangular cross section.

The temperature at the top surface decreases instantaneously to zero degrees centigrade and remains constant thereafter

$T(z=h ; t)=0^{\circ} \mathrm{C}$.

The temperature at the bottom surface of the beam, in turn, remains constant at the reference temperature

$T(z=0, t)=T_{\text {ref }}$.

All other surfaces of the beam are considered to be perfectly insulated, such that one-dimensional heat conduction along the height of the beam, i.e. along the $\mathrm{z}$-direction, takes place.

\subsection{Solution of the heat conduction problem}

One-dimensional heat conduction in $z$-direction is described by the following partial differential equation

$$
\frac{\partial T}{\partial t}=a \frac{\partial^{2} T}{\partial z^{2}},
$$

where $a$ represents the thermal diffusivity of concrete. The analytical solution of the heat conduction problem defined in Eqs. (24) - (27) reads as (Binder et al. 2018, Wang et al. 2017a)

$$
\begin{aligned}
& T(z, t)=T_{r e f}\left[\left(1-\frac{z}{h}\right)+\right. \\
& \left.\sum_{n=1}^{\infty} \frac{2(-1)^{n-1}}{n \pi} \sin \left(\frac{n \pi z}{h}\right) \exp \left(-\frac{n^{2} \pi^{2} a t}{h^{2}}\right)\right] .
\end{aligned}
$$

Distributions of the temperature along the height of the beam are shown in Figure 5, for specific values of the dimensionless time instant $a t / h^{2}$. The temperature distribution along the height of the beam during the instationary heat conduction is nonlinear. Specifically, large temperature

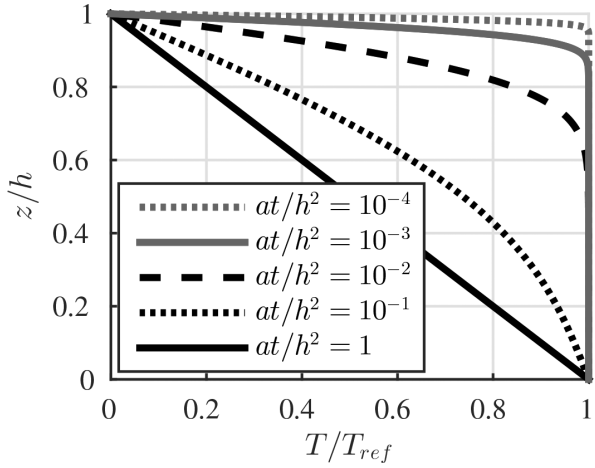

Figure 5. Distributions of the temperature along the height of the beam for different values of the dimensionless time parameter $a t / h^{2}$.

gradients are observed close to the top surface, where the sudden cooling is applied. Steady-state heat conduction has been already reached at the dimensionless time instant $a t / h^{2}=1$, resulting in a linear distribution of the temperature field along the height of the beam.

\subsection{Macrscopic thermal stress analysis based on finite element simulations}

A three-dimensional Finite Element model of a simply-supported beam, with the dimensions $l \times h \times b=2 \mathrm{~m} \times 0.3 \mathrm{~m} \times 0.4 \mathrm{~m}$, is established for macroscopic thermo-mechanical analysis. The basis for two different types of input is as follows:

- Knowledge of the volume fractions, elastic properties, and thermal expansion coefficients of the cement paste and the aggregates gives access to the corresponding thermo-mechanical properties of the concrete following Eqs. (16) and (19), see also Table 3.

- Temperature distributions at the dimensionless time instants at / $h^{2} \in\left[10^{-2}, 10^{-1}, 1\right]$, are calculated according to Eq. (28). Thereby, the reference temperature, $T_{r e f}$, and the thermal diffusivity of concrete, $a$, are set equal to $20{ }^{\circ} \mathrm{C}$ and to $4.73 \times 10^{-7} \mathrm{~m}^{2} / \mathrm{s}$, respectively.

Simulation results are the distributions of the normal stress along the height of the cross section, $\Sigma_{x x}(z)$, at midspan of the beam, see Figure 2. Right after the sudden cooling, significant tensile stresses occur at and in the vicinity of the top and the bottom surface of the beam, while compressive stresses prevail at the middle part of the beam. Notably, because of the overall equilibrium of the structure, in the absence of mechanical loads, the stress resultants, in the form of the normal forces and the bending moments, vanish at all times. 
Table 3. Thermoelastic properties of the cement paste, the aggregates, and the homogenized concrete.

\begin{tabular}{lllll}
\hline Material & Volume fraction & Elastic modulus & Poisson's ratio & Thermal expansion coefficient \\
\hline cement paste & 0.33 & $18 \mathrm{GPa}$ & 0.2 & $15.0 \cdot 10^{-6} /{ }^{\circ} \mathrm{C}$ \\
Aggregates & 0.67 & $40 \mathrm{GPa}$ & 0.2 & $8.5 \cdot 10^{-6} /{ }^{\circ} \mathrm{C}$ \\
concrete & - & $30 \mathrm{GPa}$ & 0.2 & $10.0 \cdot 10^{-6} /{ }^{\circ} \mathrm{C}$ \\
\hline
\end{tabular}

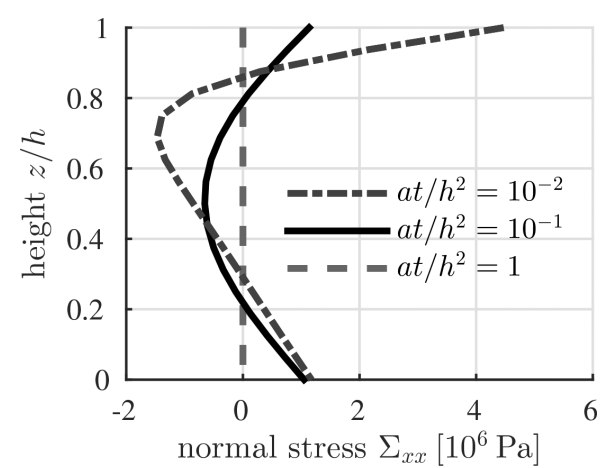

Figure 6. Evolution of the distribution of the macroscopic normal stress $\Sigma_{x x}$ along the height of the crosssection at the middle length of the beam with heat conduction.

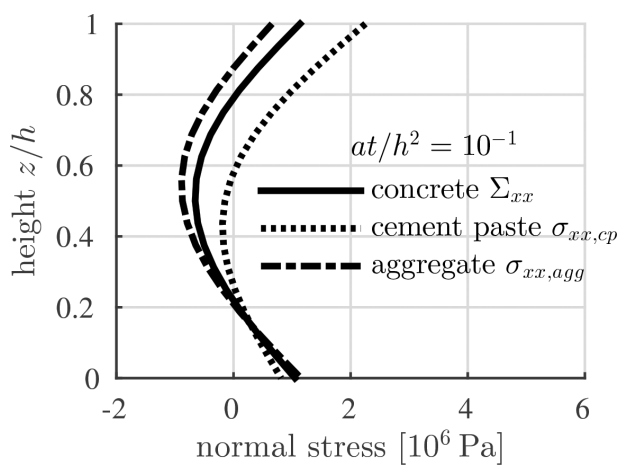

Figure 7. Distribution of the macroscopic stresses of concrete and of the microscopic stresses of the cement paste and the aggregates along the height of the cross section at midspan of the beam at the dimensionless time instant at $/ h^{2}=10^{-1}$.

The temperature-induced stresses are gradually decreasing. Finally they vanish as the temperature profile along the height of the beam approaches the linear distribution which refers to the steadystate heat conduction.

\subsection{Top-down quantification of stress states in the cement paste and the aggregates}

The microscopic stresses in the cement paste and the aggregates are quantified on the basis of the presented multiscale model. The calculated macroscopic stress states of concrete and the temperature distribution along the height of the beam serve as input. The microscopic stresses in the cement paste matrix and in the aggregate inclusions at the dimensionless time instant $a t / h^{2}=10^{-1}$ are shown in Figure 3. Because of the mismatch of the thermal expansion of the cement paste and the aggregates, the tensile stresses prevailing within the cement paste are larger in comparison to those within the aggregates and the concrete.

\section{SUMMARY AND CONCLUSIONS}

A multiscale thermo-hygro-poro-mechanical model for concrete was developed and applied to the multiscale structural analysis of a concrete beam.

- Bottom-up homogenization was used to upscale hygrothermic coefficients to the thermal expansion coefficients of the cement paste and the concrete.

- The homogenized stiffness and the thermal expansion coefficient were used as input for a three dimensional Finite Element simulation of a simply supported concrete beam subjected to sudden cooling at the top surface.

- Top-down stress concentration was used to quantify the microscopic stresses of the cement paste matrix and the aggregates.

This analysis has led to the following conclusions:

- The homogenization approach for thermal expansion coefficients was exemplarily validated in Subsection 2.5, where measured hygrothermic coefficients were upscaled to effective thermal expansion coefficients of different mature cement paste. The model-predicted thermal expansion coefficients agree very well with the measured counterparts. This is strong evidence of the predictive capabilities of the presented multiscale model.

- The overall multiscale structural analysis has shown that considerable macroscopic thermal stresses arise right after sudden cooling. These stresses gradually decrease and finally vanish, once heat conduction has reached the steadystate regime.

- Top-down stress concentration was carried out explicitly considering the mismatch of the 
thermal expansion coefficients of the cement paste and the aggregates. This way, it could be shown that the tensile stresses in the cement paste are significantly larger than the ones at the concrete scale. This proves that the risk of cracking of concrete is larger than estimated on the basis of the tensile stresses quantified at the macroscopic scale of concrete.

\section{ACKNOWLEDGEMENTS}

Financial support by the Austrian Science Fund (FWF), provided within project P 281 31-N32 "Bridging the Gap by Means of Multiscale Structural Analyses" is gratefully acknowledged. The first author also gratefully acknowledges financial support by the China Scholarship Council (CSC).

\section{REFERENCES}

Badmann, R., N. Stockhausen, \& M.J. Setzer (1981). The statistical thickness and the chemical potential of adsorbed water films. Journal of Colloid and Interface Science 82(2), 534-542.

Benveniste, Y. (1987). A new approach to the application of Mori-Tanaka's theory in composite materials. Mechanics of Materials 6(2), 147-157.

Binder, E., H. Wang, T. Schlappal, J. Zhang, Y. Yuan, B. Pichler, \& H. Mang (2018). Bridging the gap between concrete microstructures and tunnel linings. In Advances in Computational Plasticity, pp. 23-44. Springer.

Emanuel, J.H. \& J.L. Hulsey (1977). Prediction of the thermal coefficient of expansion of concrete. Journal of the American Concrete Institute 74(4), 149-155.

Grasley, Z.C. \& D.A. Lange (2007). Thermal dilation and internal relative humidity of hardened cement paste. Materials and Structures 40(3), 311-317.

Gudmundsson, A. (2011). Rock fractures in geological processes. Cambridge University Press.

Hill, R. (1963). Elastic properties of reinforced solids. Journal of Mechanics and Physics of Solids 11(5), 357-372.
Huang, Q., Z. Jiang, X. Gu, W. Zhang, \& B. Guo (2015). Numerical simulation of moisture transport in concrete based on a pore size distribution model. Cement and Concrete Research 67, 31-43.

Levin, V. (1967). Coefficients of temperature expansion of heterogeneous materials. Mechanics of Solids 2, $88-94$.

Mori, T. \& K. Tanaka (1973). Average stress in matrix and average elastic energy of materials with misfitting inclusions. Acta Metallurgica 21(5), 571-574.

Neimark, A.V. \& P.I. Ravikovitch (2001). Capillary condensation in MMS and pore structure characterization. Microporous and Mesoporous Materials 44, 697-707.

Pichler, B. \& C. Hellmich (2011). Upscaling quasi-brittle strength of cement paste and mortar: A multi-scale engineering mechanics model. Cement and Concrete Research 41(5), 467-476.

Pichler, B. \& L. Dormieux (2010). Cracking risk of partially saturated porous media-Part I: Microporoelasticity model. International Journal for Numerical and Analytical Methods in Geomechanics 34(2), 135-157.

Ulm, F.J., G. Constantinides, \& F. Heukamp (2004). Is concrete a poromechanics materials? - A multiscale investigation of poroelastic properties. Materials and Structures 37(1), 43-58.

Wang, H., C. Hellmich, Y. Yuan, H. Mang, \& B. Pichler (submitted). Does reversible water uptake/release by hydrates govern the thermal expansion of cement paste? - A scale transition analysis. Cement and Concrete Research.

Wang, H., H. Mang, Y. Yuan, \& B. Pichler (2017b). Microporomechanical modeling of thermal expansion of cement pastes. In Poromechanics 2017 - Proceedings of the 6th Biot Conference on Poromechanics, Paris, France, pp. 746-753.

Wang, H., M. Ausweger, H. Mang, Y. Yuan, \& B. Pichler (2017a). Multiscale quantification of thermal stresses in concrete linings resulting from accidental sudden heating or cooling. In Proceedings of the IV International Conference on Computational Methods in Tunneling and Subsurface Engineering, Innsbruck, Austria, pp. 781-788.

Zaoui, A. (2002). Continuum micromechanics: Survey. Journal of Engineering Mechanics (ASCE) 128(8), 808-816. 